worden ist. Es gibt aber sowohl für die lokalen als auch für die internationalen Akteure keine Alternativen zur Suche nach diesem Punkt, zum langwierigen Rekonstruktionsprozess der bosnischen Gesellschaft - zum bereits eingeschlagenen europäischen Weg.

Die Daytoner »Zwangsjacke« kann auf dem Weg kontinuierlicher und schon begonnener Reformen im EU-Integrationskontext »step by step « abgelegt werden. Dazu bedarf es gerade seitens der EU Visionen; es bedarf der Bereitschaft und Arbeit an der Vision eines modernen, demokratischen, auf dem Konzept einer Zivilgesellschaft beruhenden, europäischen Bosnien-Herzegowina. Sowohl in Bosnien-Herzegowina als auch in anderen Staaten des Westbalkans setzte die EU aber bis jetzt zu sehr auf bürokratische Aspekte der Entwicklung, vertraute zu sehr einer institutionellen und technokratischen Logik. Das vorläufige Scheitern der EU-Verfassung und die daraufhin in einigen EU-Staaten erhobenen Forderungen nach einer Verlangsamung der künftigen Erweiterung der Union zeigen nicht nur die internen Probleme und Grenzen der EU auf, sondern wirkten sich geradezu negativ auf jene Nachbarregion aus, deren volle Integration sowohl der Region selbst als auch der EU nutzen würde.

Die EU muss daher gerade in den nächsten Monaten durch eine aktive, mutige und gestalterische Politik auf dem Balkan abseits diplomatischer Floskeln und leerer Versprechungen zeigen, dass ihr die südosteuropäischen Nachbarn wichtig sind. Mehr Aufmerksamkeit, größere Anstrengungen, konkrete und an Bosnien-Herzegowina und den Balkan angepasste Strategien und finanzielle Mittel aus Brüssel werden vor allem 2006 entscheidend sein, um für die Lösung der anstehenden Probleme stabile Rahmenvoraussetzungen zu schaffen. Die Strategie muss auch der Tatsache Rechnung tragen, dass ohne eine verbindliche Einbindung der lokalen Entscheidungsträger in die Entscheidungen Fortschritte nur sehr langsam zu erreichen sein werden. Die Region kann keine »Diktate von oben« brauchen, vielmehr braucht sie gleichberechtigte und konstruktive Partner.

Daher müssen auch die bosnischen Behörden und Bürger viel stärker und entschiedener als bislang ihr Schicksal in die eigenen Hände nehmen und offensiver an der Umsetzung der notwendigen Reformen auf dem europäischen Weg arbeiten. Dazu bedarf es neuer, junger und nicht-nationalistischer Akteure, die mit einem neuen Selbstbewusstsein und in Partnerschaft mit der internationalen Gemeinschaft den Reformprozess gestalten. Eines muss dabei klar sein: Dayton und Brüssel sind nicht kompatibel!

\title{
Das Kosovo und die Statusfrage Rechtliche Reflexionen und politische Optionen
}

\author{
Alexander S. Neu*
}

Abstract: Six years after the UN-Protectorate on Kosovo (UNMIK) has been established, the international community seems to feel an exhaustion in regard to Kosovo. New crises such as international terrorism emerged and demand a shift of the political and military attention. Solving the outstanding issue of the status of the Kosovo became an urgent necessity. Despite the need for a quick settling, the international community is still struggling for a consensus on how to deal with the uncompromising stance between Serbia and the Kosovo Albanians: should Kosovo remain part of Serbia or gain independence? Based on an analysis of the legal issues, like international law and constitutional law, this essay outlines the political options available to the international community.

Keywords: Kosovo, Rechtsfragen, politische Optionen, internationale Organisationen

I m Oktober 2005 entschied der UN-Sicherheitsrat, den Weg für Verhandlungen zur Klärung des endgültigen rechtlichen Status der serbischen Provinz freizugeben. Die so genannte Balkan-Kontaktgruppe ist die einflussreichste Kraft bei den anstehenden Verhandlungen. Sie besteht aus Frankreich, Russland, Deutschland, Großbritannien, Italien und den USA. Die Kontaktgruppe ist eine Fortführung der Bosnien-Kontaktgruppe, die sich während des Bosnienkrieges Anfang der neunziger Jahre gebildet hatte. Als externer Akteur erhebt sie einen Re-

\footnotetext{
* Dr. phil. Alexander S. Neu, Journalist und wissenschaftlicher Mitarbeiter am
} Berliner Informationszentrum für Transatlantische Sicherheit e.V., Berlin. gulierungs- und Gestaltungsanspruch für den gesamten Westbalkan. Der UN-Sicherheitsrat soll schließlich das letzte Wort haben und die angestrebte Lösung völkerrechtlich absegnen.

All dem vorausgegangen waren die gewaltsamen Übergriffe von Kosovo-Albanern gegen im Kosovo lebende Serben im Jahre 2004. Seitdem stand die serbische Provinz Kosovo wieder verstärkt auf der internationalen Agenda. Die März-Unruhen 2004 werden vor allem von den Protagonisten der staatlichen Unabhängigkeit als Nachweis dafür gesehen, dass diese zwingend notwendig sei, um Stabilität in der Region zu erreichen. 
Bemerkenswert ist, dass diese Kräfte es vermocht haben, die so genannte »Statusfrage« zum Gegenstand einer völkerrechtlichen Betrachtung zu machen, obgleich dies vor dem Hintergrund der eindeutig definierten Resolution 1244, die unmissverständlich nur eine staatsrechtliche Lösung zulässt, gar nicht Gegenstand der Verhandlungen sein kann.

\section{Die Republik Serbien und ihre Provinz Kosovo}

Die serbische Provinz Kosovo ist historisch und verfassungsrechtlich ein fester Bestandteil der Republik Serbien. Eine staatliche Unabhängigkeit des Kosovo käme einer Teilung der Republik Serbien gleich. ${ }^{1}$ Die Teilung der Republik Serbien wäre vergleichbar mit der Teilung der Republik Bosnien-Herzegowina oder Kroatiens, was die internationale Staatengemeinschaft unter Anwendung von Waffengewalt seinerzeit verhinderte.

Mit der Anerkennung der Unabhängigkeit des Kosovo würde die bislang mit Blick auf Jugoslawien als oberste Maxime praktizierte Politik der territorialen Integrität der jugoslawischen Nachfolgerepubliken gebrochen. Die daraus entstehende politische und völkerrechtliche Inkonsistenz und Opportunität ist nicht nur im Hinblick auf die künftige Statusfrage des Kosovo, sondern angesichts weltweit bestehender Unabhängigkeitsbestrebungen für die künftige völkerrechtliche Praxis generell von entscheidender Bedeutung. Um die Problematik in ihrer Komplexität verständlich zu machen, ist es zunächst erforderlich, den genauen verfassungsrechtlichen Status des Kosovo zu analysieren: Das Recht der externen Selbstbestimmung wurde in der jugoslawischen Verfassung lediglich den sechs jugoslawischen Republiken zuteil, nicht jedoch den beiden serbischen Provinzen Kosovo und Vojvodina.

Als Träger der staatlichen Souveränität wurden die einzelnen Völker (Nationen) sowie die »Arbeiterklasse und alle Werktätigen « definiert: »Die sozialistische Republik ist ein auf der Souveränität des Volkes und auf der Macht und der Selbstverwaltung der Arbeiterklasse und aller Werktätigen beruhender Staat. $\ll^{2}$

Als Volk anerkannt wurden die Slowenen, Kroaten, Serben, bosnischen Muslime, Montenegriner und Makedonier. Die übrigen Volksgruppen erhielten den Status von »Völkerschaften«(Nationalitäten), der als Status für anerkannte Minderheiten zu bewerten ist. Der Zweck dieser auf den ersten Blick seltsam anmutenden Differenzierung zeigt sich bei näherer Betrachtung. Es handelte sich um eine Vorkehrung, die Grenzverschiebungen sowohl mit Blick auf den jugoslawischen Gesamtstaat als auch mit Blick auf die Republik Serbien verhindern sollte. Die beiden größten Ethnien Jugoslawiens, denen lediglich der Status einer Nationalität zuerkannt wurde, leben größtenteils in unmittelbarer Nachbarschaft zu ihren Mutterländern (Albanien und Ungarn), so dass die Furcht vor Vereinigungsforderungen groß war und ist. Vor diesem Hintergrund wurden diesen Regionen zwar weitgehende Autonomierechte (Provinzstatus) innerhalb Serbiens - sogar mit pa-

1 Verfassung der SFR Jugoslawien - Erster Teil - Die Sozialistische Föderative Republik Jugoslawien - Artikel 1, 4, S. 9 f.

2 Ebd., S. 92 f. ritätischen Beteiligungsrechten auf Bundesebene - zuerkannt, jedoch der Republikstatus aufgrund des damit verbundenen Sezessionsrechts nicht verliehen.

Mit dem Terminus »Provinz« wurden zwei wesentliche rechtliche Unterschiede zu den Republiken gezogen: Erstens kam den Provinzen keine Staatlichkeit zu, da der Terminus Republik eine Rechtskategorie darstellte, die die Möglichkeit des externen Selbstbestimmungsrechts implizierte. Und zweitens wurden die Nationalitäten nicht einmal als Träger der Autonomie anerkannt. Lediglich die »Arbeiterklasse und alle Werktätigen «, also eine soziale Klasse, wurde als Träger der autonomen Rechte, die den Provinzen zuerkannt wurden, definiert: »Die sozialistische Provinz ist eine auf der Macht der Arbeiterklasse und aller Werktätigen beruhende autonome, sozialistische, selbstverwaltende, demokratische gesellschaftlich-politische Gemeinschaft. «3

Somit ist die oft zu vernehmende Aussage, die Albaner hätten Autonomierechte gehabt, rechtlich ebenso unzutreffend wie die Behauptung, sie hätten auch oder zumindest quasi einen Republikstatus genossen. Lediglich der »Arbeiterklasse und allen Werktätigen « der geographischen Region Kosovo - und das waren nahezu alle Einwohner ungeachtet ihrer Nationalität und somit auch die Kosovo-Serben - wurde eine Autonomie innerhalb der Republik Serbien eingeräumt.

\section{Der völkerrechtliche Status der serbischen Provinz Kosovo}

Die den NATO-Krieg 1999 beendende UN-Resolution 1244 stellt unzweideutig fest, dass das Kosovo ein Bestandteil der Bundesrepublik Jugoslawien ist: »[...] Kosovo kann eine substantielle Autonomie innerhalb der Bundesrepublik Jugoslawien genießen $[\ldots] \ll .4$

Die Resolution spiegelt das Ergebnis des Krieges von 1999 wider, den die Bundesrepublik Jugoslawien zwar verlor, aus dem die NATO jedoch auch nicht als eindeutiger Sieger hervorging. Die Resolution 1244 bestätigt die UN-Sicherheitsratsresolution 1199 aus dem Jahre 1998, die verabschiedet wurde, als Belgrad noch die Hoheitsgewalt über das Kosovo ausübte. In dieser Resolution wurde ebenfalls das »Bekenntnis aller Mitgliedstaaten zur Souveränität und territorialen Unversehrtheit der Bundesrepublik Jugoslawien« bekräftigt. Der Sicherheitsrat unterstützte bereits hier »einen erhöhten Status für Kosovo, eine substantielle Autonomie und eine bedeutsame Selbstverwaltung des Kosovo «. Nichts anderes besagt eine weitere UNSicherheitsratsresolution (1345) aus dem Jahre 2001, als die Provinz bereits nahezu zwei Jahre von der UNMIK verwaltet wurde. Auch in diesem Beschluss bekräftigte der Sicherheitsrat seine »Verpflichtung zur Souveränität and territorialen Unversehrtheit der Bundesrepublik [...] wie in der Helsinki-Schlußakte ausgeführt.$^{5}$

\footnotetext{
3 Ebd., Artikel 4.

4 Resolution 1244 (1999).

5 Resolution 1199 (1998), adopted by the Security Council at its 3930th meeting on September 1998, <http://daccessdds.un.org/doc/UNDOC/GEN/N98/279/96/ PDF/N9827996.pdf?OpenElement>; Resolution 1345 (2001), adopted by the Security Council at its 4301st meeting, on 21 March 2001, <http://daccessdds. un.org/doc/UNDOC/GEN/N01/298/89/PDF/N0129889.pdf?OpenElement>.
} 
Angesichts der politischen Entwicklung von 1998 bis heute kommt hier eine Kontinuität im Hinblick auf den Status des Kosovo in Jugoslawien und in der Republik Serbien zum Ausdruck. Diese Haltung scheint nicht nur im UN-Sicherheitsrat vorzuherrschen, sondern war auch zwischen den ehemaligen Kriegsparteien, der NATO und der BR Jugoslawien, Konsens: Im Military Technical Agreement, das die Kriegsparteien am 9. Juni 1999 zur Beendigung der Kämpfe unterzeichneten, wird das Kosovo mehrfach indirekt als Teil der Bundesrepublik Jugoslawien (»[...] Kosovo Provinzgrenze in den Rest des Territoriums der Bundesrepublik Jugoslawien ${ }^{6}$ « sowie »[...] Kontrolle der Grenzen der Bundesrepublik Jugoslawien in Kosovo mit Albanien und Makedonien [...]«) und sogar der Republik Serbien (»[...] zu Orten in Serbien außerhalb Kosovo«) genannt. ${ }^{7}$ Selbst die Statusänderung der BR Jugoslawien hin zu der Staatenunion Serbien-Montenegro ändert nichts an der Souveränität Belgrads über das Kosovo, da die Staatenunion sich als völkerrechtlicher Rechtsnachfolger der BR Jugoslawien mit einheitlicher Rechtspersönlichkeit betrachtet und so auch von der internationalen Staatengemeinschaft akzeptiert wird.

Auch wenn Montenegro den Staatenbund verlassen sollte, so würde verfassungsrechtlich Serbien die Souveränität über das Kosovo beibehalten. Bereits in der Präambel der Verfassungsurkunde wird die territoriale Zugehörigkeit des Kosovo zu Serbien bekräftigt: »[...] der Staat Serbien, welcher die autonome Provinz Vojvodina und die autonome Provinz Kosovo und Metohija einschließt [...].«

Darüber hinaus und vorsichtshalber mit Blick auf das Kosovo wird im Falle des Austritts Montenegros aus der Staatenunion die alleinige völkerrechtliche Rechtsnachfolge Serbien zu erkannt: »[...] Sollte Montenegro aus der Staatenunion Serbien und Montenegro austreten, würden die internationalen Instrumente [Angelegenheiten], die für die Bundesrepublik Jugoslawien von Bedeutung waren, insbesondere UN Sicherheitsratsbeschluss 1244, in ihrer Gesamtheit auf Serbien als Rechtsnachfolger übergehen. $\ll^{8}$

Angesichts dessen kann unter juristischem Aspekt - nehmen die Mitglieder des UN-Sicherheitsrates ihre eigenen Resolutionen und die UNO ernst - der Gegenstand der Verhandlungen über die Statusfrage nur staatsrechtlicher, nicht indes völkerrechtlicher Natur sein. Mit anderen Worten: Nicht die staatliche Unabhängigkeit steht zur Diskussion, sondern lediglich die konkrete Ausgestaltung einer substantiellen Autonomie kann Gegenstand der Verhandlungen sein. Was aber kann eine für alle Seiten akzeptable und zuletzt auch tragfähige Lösung der Kosovofrage sein?

\section{Lösungsmodelle}

Eine sinnvolle und tragfähige Lösung muss einerseits den politischen Realitäten und Interessen und andererseits den völker- und verfassungsrechtlichen Grundlagen gerecht werden,

\footnotetext{
6 FRY: Federal Republic of Yugoslavia

7 Military Technical Agreement, Art. I. und II.

8 Constitutional Charter of the State Union of Serbia and Montenegro, 2003, Präambel und Art. 60, S. 10.
}

so dass das Modell nicht zu dem allseits gefürchteten völkerrechtlichen Präzedenzfall wird. Nur so ist zum einen der Vorbehalt jener Staaten im UN-Sicherheitsrat, die mit ähnlichen nationalpolitischen Konflikten konfrontiert sind, zu überwinden, und zum anderen der Präzedenzfall zu verhindern, auf den sich andere nationale Bewegungen berufen könnten. Vier Modelle lassen sich diskutieren:

\subsection{Das Preševo-Modell}

Das Preševo-Tal, das von den Albanern als »Ost-Kosovo« bezeichnet wird, liegt östlich der serbischen Provinz Kosovo und ist Bestandteil Zentralserbiens, d.h. Serbien ohne seine Provinz Kosovo. In dieser Region leben ca. 70.000 Albaner. In den Jahren 2000/2001 hatte sich dort ein UÇK-Ableger breit gemacht und terroristische Anschläge verübt. Ähnlich wie im Kosovo zielte die UÇK darauf ab, das Eingreifen des Westens zu erzwingen. ${ }^{9}$ Der Westen machte diesmal indessen keine Anstalten, dem Manöver der UÇK zu folgen.

Stattdessen verabschiedete der UN-Sicherheitsrat eine Resolution, in der die Aktivitäten der albanischen Seite mit scharfen Worten abgelehnt wurden. ${ }^{10}$ Zusätzlich wurde den jugoslawischen Sicherheitskräften erlaubt, in der Pufferzone auch mit militärischen Mitteln die staatliche Ordnung wiederherzustellen. Die Operation verlief unproblematisch; eine OSZEMission begleitete die Transformation der Gemeindeverwaltung zugunsten multiethnischer Strukturen. ${ }^{11}$ Seitdem ist das Preševo-Tal im Wesentlichen befriedet.

Die Frage ist, ob sich dieses Modell auch auf die Provinz anwenden ließe. Das Modell hätte eine reale Chance gehabt - wenn der Westen von Anfang an, also zu Beginn der Kosovo-Krise, Belgrad diplomatisch darin unterstützt hätte, einen besseren Weg zu finden, den Konflikt in der Region beizulegen, anstatt zugunsten der kosovo-albanischen Seite Partei zu ergreifen, Ultimaten zu formulieren und diese unter Androhung und Durchführung militärischer Maßnahmen zu unterstreichen. Letztlich bekamen kosovo-albanische Separationsforderungen erst durch die Parteinahme der NATO den entsprechenden Auftrieb. ${ }^{12}$ Selbst nach Beendigung des Krieges und der Errichtung des UN-Protektorats wäre eine »Preševo-nahe Lösung « möglich gewesen, wenn die UNMIK die Resolution 1244 textnah interpretiert und implementiert hätte.

Ein feste Position gegenüber beiden Konfliktparteien und nicht nur gegenüber Serbien oder den Kosovo-Serben hätte wesentlich mehr Druck auf die kosovo-albanische Seite ausgeübt, mit Belgrad über die konkrete Ausgestaltung substantieller Autonomierechte zu verhandeln. Da allerdings die UNMIK nichts unversucht gelassen hat, um den kosovo-albanischen Wünschen zu entsprechen, ist es problematisch, bereits gewährte Rechte wieder zurückzunehmen.

\footnotetext{
9 Die Politik der UÇK bedroht die bestehenden Grenzen, in: Le Monde diplomatique, 12.4.2001.

10 UNO-Resolution 1345 (2001)

11 OSCE Press Release, »OSCE welcomes constitution of municipal assembly of Bujanovac«, Belgrade 16.9.2002, (http://www.osce.org/item/6979.html); Konfliktbarometer (HIIK), Jugoslawien, http://www.hiik.de/de/barometer2001/texte/jugoslawien.htm.

12 Heinz Loquai: Der Kosovo-Konflikt - Wege in einen vermeidbaren Krieg - Die Zeit von Ende November 1997 bis März 1999, Baden-Baden 2000.
} 


\subsection{Das Junktim-Modell}

Das Junktim-Modell hat eine rein staatsrechtliche Dimension und verbindet zwei Ebenen,

- die Republik Serbien und ihre Provinz Kosovo;

- die Provinz Kosovo und ihre Enklaven sowie das nahezu ausschließlich serbisch bewohnte Nordkosovo.

Das Junktim-Modell verbindet die an die Kosovo-Albaner zu verleihenden Autonomierechte mit den Autonomierechten, die den Kosovo-Serben zu gewähren sind. Das Junktim-Modell setzt dort an, wo die Verantwortlichen in Pristina und Belgrad die Entscheidung in gegenseitigem Einvernehmen durch Verhandlungen herbeiführen müssten. Dieser Ansatz beinhaltet zudem einen pädagogischen Aspekt: Beide Seiten sind gezwungen, miteinander und verantwortlich eine Lösung zu finden, die für diese Region, in der beide Volksgruppen leben, eine nachhaltige Stabilität erzeugt. Die internationale Staatengemeinschaft wäre nur für die Überwachung der Umsetzung verantwortlich.

\subsection{Das Modell des begrenzten südwestlichen Territorialverlustes Serbiens}

Das Modell des begrenzten südwestlichen Territorialverlustes stellt die konsequente Fortsetzung des Junktim-Modells dar für den Fall, dass die kosovo-albanische Seite sich mit substantieller Autonomie im Rahmen Serbiens nicht anzufreunden vermag. Die bisherigen Verwaltungsgrenzen dürfen nicht als sakrosankt betrachtet werden. Um dies verständlicher zu machen, bedarf es eines Perspektivwechsels. Von der Annahme eines multiethnisch einheitlich existierenden Kosovo ist Abschied zu nehmen: Nicht das Kosovo wird geteilt, da es ohnehin im Rahmen der jugoslawischen Verfassung keine Staatlichkeit besaß, geteilt würde vielmehr Serbien. Die Frage ist, ob Serbien den gesamten Südwesten seines Staatsgebietes (29 Gemeinden ${ }^{13}$ ) verliert oder nur den größten Teil (26 Gemeinden).

Das mantrahaft wiederholte Argument, die bestehenden Grenzen müssten aufrechterhalten werden, kann angesichts der internationalen Handhabung der Desintegration der jugoslawischen Föderation, des Umgangs mit der Republik Serbien und seiner Provinz Kosovo sowie der nun vermutlich auch zur Disposition gestellten Gemeindegrenzen im Kosovo und der veränderten Gemeindegrenzen in Makedonien ${ }^{14}$ zugunsten der dortigen albanischen Bevölkerung nicht überzeugen. Es ist auffällig, dass das Uti-possidetis-Prinzip dann dem eigentlich nur sekundären, d.h. im Notfall anzuwendenden, externen Selbstbestimmungsrecht untergeordnet wird, wenn die territoriale Integrität und Souveränität des jugoslawischen Gesamtstaates und der Republik Serbien zur Diskussion gestellt werden. Dasselbe Prinzip wird hingegen als prioritäre, ja unausweichliche Option behauptet, wenn es um die territoriale Mitnahme - ungeachtet darin lebender Minderheiten - der

\footnotetext{
13 Die OSZE-Homepage spricht fälschlicherweise von 30 Gemeinden, zählt dann aber nur 29 auf, <http://www.osce.org/kosovo/13985.html>.

14 Informationen zur Deutschen Außenpolitik, »Entweder oder«, 7.11.2004, http://www.german-foreign-policy.com/de/news/art/2004/47472.php.
}

jugoslawischen Erbmasse der nicht-serbischen Volksgruppen geht.

Vor diesem Hintergrund ist die Argumentation, mit einer Teilung des Kosovo und der damit einhergehenden Grenzveränderung würde man einen Präzedenzfall schaffen, ein rein politisch-opportunistisch motiviertes Argument, da man in Wirklichkeit eine politische und unter Umständen gewaltsame Konfrontation mit radikalen Kosovo-Albanern fürchtet. Ebenso wenig ist die These einer ethnischen Kettenreaktion haltbar: Erstens hat eine solche Kettenreaktion bereits auf dem gesamten Gebiet des ehemaligen Jugoslawien stattgefunden und wurde vom Westen nachträglich weitgehend anerkannt. Zweitens hat die internationale Staatengemeinschaft in den Jahren 2000 und 2001 im Presevo-Tal und in Makedonien gemeinsam mit den Regierungen in Belgrad und Skopje durch entschiedenes Handeln schließlich der ethnischen Kettenreaktion ein Ende gesetzt. ${ }^{15}$

\subsection{Das Modell einer konditionierten Unabhän- gigkeit mit EU-Perspektive}

Folgt man gegenwärtigen Diskussionen, etwa den Überlegungen der »International Commission on the Balkans«, so wird eine konditionierte Unabhängigkeit eines ungeteilten Kosovo mit europäischer Perspektive favorisiert. ${ }^{16}$ Was diese jedoch beinhalten soll, bleibt weitgehend unklar. Die konditionierte Unabhängigkeit soll temporär sein und in eine EU-Mitgliedschaft münden. Mit anderen Worten: In dem Moment, in dem das konditionale Moment aufgehoben würde und das Kosovo theoretisch die uneingeschränkte Unabhängigkeit besäße, würde mit dem Beitritt zur EU genau diese politische Unabhängigkeit wieder eingeschränkt werden. Auf diese Weise würde das Kosovo zwar einen souveränen, aber eben keinen unabhängigen Staat darstellen, da es ein erhebliches Maß an politischen Kompetenzen an die EU delegieren müsste.

Wie dies angesichts der Tatsache, dass das Kosovo die ärmste und unberechenbarste Region Europas ist und dies auf unabsehbare Zeit bleiben wird, politische Realität werden soll, bleibt unklar. Wenig überzeugend an dem Bericht der »International Commission on the Balkans « sind die Äußerungen zu den Minderheitenrechten. Wie will die internationale Gemeinschaft nicht nur die geschriebenen, sondern vor allem die gelebten Rechte garantieren? Wie will die internationale Staatengemeinschaft eine schleichende Vertreibung und Verdrängung der nicht-albanischen Bevölkerungsteile verhindern?

Hierzu favorisiert die »International Commission on the Balkans « zwar eine Dezentralisierung des Kosovo, was eine Übertragung möglichst vieler Verwaltungskompetenzen von Pristina auf die Gemeinden bedeutet. Dieser an sich begrüßenswerte Ansatz ist allerdings wiederum mit einer Konzession an die albanische Seite verbunden und höhlt damit das

15 Ebd.

16 Report of the International Commission on the Balkans: The Balkans in Europe's Future, Sofia [Centre for Liberal Strategies]. April 2005, S. 19 ff., www.balkan-commission.org/activities/Report.pdf. 
eigentliche Ziel wieder aus, diese Volksgruppen zu schützen. Gefordert wird eine Dezentralisierung mit einer Reform der Gemeindegrenzen, so dass es keine rein serbischen Gemeinden geben soll. ${ }^{17}$ Legitimiert wird dieser Ansatz durch die immer noch aufrechterhaltene, jedoch empirisch widerlegte, Vorstellung von der Existenz einer multiethnischen kosovarischen Gesellschaft. ${ }^{18}$

Eine Untersuchung der International Commission on the Balkans zeigt ungewohnt offen die erschreckende Tatsache, dass 72 Prozent der befragten Menschen in Kosovo und 68 Prozent in Albanien einen ethnisch »reinen « Staat bevorzugen. Diese Auffassung teilen in Serbien vergleichsweise wenige (19 Prozent) und selbst in Bosnien-Herzegowina »nur « 29 Prozent. ${ }^{19}$ Das mittelfristige und langfristige Resultat eines Konzeptes, das darauf zielt, die Gemeindegrenzen zu verändern, wird die Verdrängung der Serben und anderer Volksgruppen aus den lokalen Verwaltungsstrukturen und infolgedessen aus dem Kosovo sein.

Was die Skepsis gegenüber dem »konditionierten Unabhängigkeitsmodell« und den darin erwähnten Minderheitenrechten begründet, ist die Frage, woher die Befürworter dieses Modells den Optimismus für den Erfolg eines multiethnischen Kosovo nehmen. Denn bislang hat die internationale Staatengemeinschaft in Gestalt der UNMIK und KFOR dies nicht einmal ansatzweise vermocht. Im Gegenteil, sie hat 1999 der Flucht und Vertreibung von über 200.000 Nicht-Albanern tatenlos zugesehen und bis heute die Grundlagen für die Rückkehr einer nennenswerten Zahl von Betroffenen nicht geschaffen. Dieses Versagen räumt sogar der »Report of the International Commission on the Balkans « ein: "Die internationale Staatengemeinschaft hat in ihrem Versuch, Sicherheit und Entwicklung in die Provinz zu transportieren, ganz offensichtlich versagt. Ein multiethnisches Kosovo existiert nicht - außer in den bürokratischen Einschätzungen der internationalen Staatengemeinschaft. [...] Die Situation der serbischen Minderheit in Kosovo ist die größte Anklage gegen den Willen und die Fähigkeit Europas, seine eigenen proklamierten Werte zu verteidigen. $\ll^{20}$

Die konditionierte Unabhängigkeit, wie sie auch von dem Bericht der International Commission on the Balkans favorisiert wird, wirft mehr Fragen auf, als gesicherte Antworten gefunden werden können. Geht dieses Experiment schief, wofür angesichts der Erfahrungen mehr spricht als dagegen, dann sind die Opfer dieses westlichen Experiments erneut die Menschen vor Ort.

\section{Bilanz}

Diese Analyse zeigt, dass das Presevo-Modell nicht tragfähig ist. Nicht minder unrealistisch ist das konditionierte Unabhängigkeitsmodell, da es einer monoethnische Bevölkerungsstruktur

17 So Rolf Ekeus, Hoher Kommissar der OSZE für Nationale Minderheiten, auf der Veranstaltung »Der westliche Balkan. Politische Ordnung, wirtschaftliche Stabilität und internationales Engagement: Kosovo«, Berlin 2005.

18 Report of the International Commission on the Balkans, S. 19.

19 Ebd. Siehe auch Graphik 22, S. 53.

20 Ebd., S. 19. der Region Kosovo mit faktischer Duldung durch die internationale Staatengemeinschaft Tür und Tor öffnet.

Demgegenüber stellen das Junktim-Modell und das Modell des begrenzten südwestlichen Territorialverlustes realistische Modelle dar, um weiteres menschliches Leid zu verhindern und dem internationalen Recht wieder Geltung zu verschaffen. Diese Modelle weisen weitere Vorteile auf: Zunächst bleibt festzustellen, dass sich beide Varianten nicht gegenseitig ausschließen. Entweder könnte das Modell des begrenzten südwestlichen Territorialverlustes generell in den Mittelpunkt gestellt werden oder aber als ultimative Lösung Anwendung finden, wenn die kosovo-albanische Seite eine substantielle Autonomie innerhalb Serbiens nicht akzeptiert. Das birgt zahlreiche konkrete Vorteile.

Das Gefühl in der serbischen Gesellschaft, ungerecht behandelt zu werden, würde erheblich reduziert, die Gefahr einer Hinwendung zu radikalen Parteien somit merklich abnehmen. Es wäre ein Signal an die Serben, dass ihre Reformen auch wirklich anerkannt und sie nicht für die Taten Milosevics weiterhin bestraft werden würden. Hierdurch würde Serbien aufgrund seiner Größe und zentralen geographischen Lage zu einem Stabilitätsfaktor in Südosteuropa.

Ein monoethnisches Kosovo - gedacht nicht als politische Gemeinschaft, sondern als Region - würde damit verhindert. Mit der Unabhängigkeit - ungeachtet ob konditioniert oder unkonditioniert - würden Flucht, Vertreibung und schleichende Verdrängung der nichtalbanischen Ethnien nicht gestoppt, sondern geradezu herbeigeführt. Umgekehrt könnte und würde eine Vielzahl nichtalbanischer Vertriebener und Flüchtlinge in die drei nördlichen Gemeinden des Kosovo und nach Nord-Mitrovica zurückkehren und dort siedeln. Auf diese Weise könnte die internationale Gemeinschaft öffentlichkeitswirksam auf die Rückkehr einer großen Zahl Vertriebener und Flüchtlinge verweisen, die zwar nicht an ihre Heimatorte, so doch in ihre Region zurückgekehrt sind.

Darüber hinaus kann Belgrad im Rahmen des Junktim-Modells oder des Modells des begrenzten südwestlichen Territorialverlustes bis zum Ibar-Fluss die Grenze effektiver vor illegalen Grenzüberschreitungen überwachen als dies irgendwo in den unübersichtlichen Bergen hinter den Gemeinden Leposavic und Zubin Potok der Fall wäre.

Die sowohl in Nord- als auch Süd-Mitrovica arbeitenden Trepca-Werke würden von den Regierungen in Belgrad und Pristina in einer Art Joint-Venture paritätisch verwaltet werden. Der Zwang, Trepca gemeinsam zu verwalten und das beiderseitige Interesse an einem funktionierenden und gewinnbringenden Werk dürften einen gewaltigen pädagogischen Effekt für beide Seiten mit sich bringen.

Den radikalen albanischen Kräften würde damit deutlich signalisiert, dass ihre berechnenden Gewaltexzesse von 1998 bis heute nicht durch ständiges Einlenken und Parteinahme des Westens belohnt würden. Die Gewalt als bislang erfolgreiches Instrument zur Durchsetzung ihrer Ziele würde damit definitiv als nicht mehr wirksam wahrgenommen werden müssen. Auf diese Weise müssten sie lernen, ihre politischen Interessen Serbien und der ganzen Region gegenüber durch verant- 
wortungsvolle Politik und nicht durch Gewalt und Boykott zu artikulieren.

Die internationale Staatengemeinschaft würde mit beiden Modellen, die in einer Verhandlungslösung zwischen Belgrad und den Kosovo-Albanern erreicht oder auch nicht erreicht werden würde, aus dem völkerrechtlichen Dilemma herauskommen:

1. Sollten die Kosovo-Albaner eine substantielle Autonomie (Junktim-Modell) akzeptieren, wäre die Resolution 1244 erfüllt, da die Statusfrage keine völkerrechtliche Dimension bekäme, sondern eine staatsrechtliche Angelegenheit innerhalb Serbiens bliebe. Die internationale Staatengemeinschaft (hier UNO und EU) würde ihrerseits als externer Garant des Abkommens Pate stehen.

2. Sollte sich die kosovo-albanische Seite dem Junktim-Modell komplett verschließen, so würde damit der Automatismus für das Modell des begrenzten südwestlichen Territorialverlustes als Bedingung ihrer (temporär) konditionierten oder auch unkonditionierten Unabhängigkeit eintreten. Die internationale Gemeinschaft würde auf diese Weise gegenüber Belgrad ein wirkliches substantielles Entgegenkommen für dessen freiwillige Zustimmung zum Verlust des größten Teiles der serbischen Provinz signalisieren. Durch den freiwilligen Verzicht Belgrads auf den größten Teil seines südwestlichen Territoriums wäre das Faktum einer von außen aufgezwungenen Teilung der Republik Serbien nicht mehr gegeben. Das internationale Recht würde dadurch nicht weiter ad absurdum geführt. Eine unerwünschte Signalwirkung an andere Minderheiten entfiele. Ebenso würde das Selbstbestimmungsrecht der Völker in diesem Falle gleichermaßen den Kosovo-Albanern wie auch den Serben zuteil werden.

Schließlich spricht für das Junktim-Modell oder für das Modell des begrenzten südwestlichen Territorialverlustes, dass die Vereinten Nationen und die EU mit den bereits erdrückenden wirtschaftlichen, sozialen und demographischen Problemen des wesentlich größeren albanischen Teiles der Region Kosovo noch in den nächsten Dekaden in ausreichendem Maße beschäftigt sein werden.

Die Frage, ob das Modell des begrenzten südwestlichen Territorialverlustes dem Junktim-Modell vorzuziehen sei, muss sich nicht die internationale Staatengemeinschaft stellen. Die Antwort wird die kosovo-albanische Seite zu liefern haben: Entweder werden die Kosovo-Albaner für eine substantielle Autonomie Kosovos in Serbien und somit auch für eine substantielle Autonomie der serbischen Gemeinden innerhalb des Kosovo stimmen, oder sie werden ungeachtet des Preises des Verzichts auf nahezu rein serbisch besiedelte Gemeinden des nördlichen Kosovo zugunsten Zentralserbiens die Unabhängigkeit favorisieren.

Auf diese Weise müssen die Albaner akzeptieren und lernen, dass das von ihnen eingeforderte Selbstbestimmungsrecht - ungeachtet ob intern oder extern - gemäß dem Reziprozitätsprinzip ebenso den Serben zuteil werden muss. Eine Rückführung der nahezu rein serbisch bewohnten Gemeinden Zubin Potok, Zvecan, Leposavic und Nord-Mitrovica unter serbische Hoheitsgewalt stellt angesichts des unmittelbar an- grenzenden Rest-Serbiens keinerlei Probleme dar. Ferner wird sich die Frage der serbischen Enklaven im Falle einer konditionierten oder wie auch immer zu beschreibenden Unabhängigkeit so oder so mittelfristig erübrigen. Auch hier sprechen die Erfahrungen seit Sommer 1999 für sich.

\section{Fazit}

Will die internationale Gemeinschaft für alle beteiligten Akteure eine trag- und zukunftsfähige Lösung finden, so dass auf der einen Seite die gültigen internationalen Normen nicht weiter ad absurdum geführt werden und auf der anderen Seite verhindert wird, dass durch Vertreibung und Verdrängung nichtalbanischer Bevölkerungsteile eine monoethnische Region Kosovo entsteht, so muss ihre Phantasie über die bisherigen Modelle hinausreichen. Selbstgesetzte politische Tabus müssen auf ihre Realitätstauglichkeit hin überprüft und gegebenenfalls gebrochen werden, um nicht von vornherein den Handlungsspielraum aller Verhandlungsparteien unproduktiv einzuschränken. Auch die beiden Konfliktparteien müssen bereit sein, Tabus zu brechen: So sehr sich Belgrad und die Kosovo-Albaner darüber im Klaren sind, dass Belgrad kein ehrliches Interesse daran haben kann, ein Volk gegen seinen dezidierten Willen zu verwalten, so sehr sollte auch Pristina sich selbst gegenüber ehrlich sein und eingestehen, dass es kein Interesse daran haben kann, die serbische Ethnie im Norden des Kosovo gegen deren nicht weniger dezidierten Willen zu regieren. Das eingeforderte Selbstbestimmungsrecht muss dem Reziprozitätsprinzip Rechnung tragen. Das jugoslawische Verfassungsrecht, die Verfassungsurkunde der Staatenunion Serbien und Montenegro und das internationale Recht bieten diese Möglichkeit. Hingegen wird das Problem nicht gelöst, wenn eine alte Minderheitenproblematik durch eine neue ersetzt wird. Dies schafft vielmehr neues Leid. Die Minimierung des Leidens der betroffenen Menschen, über die entschieden wird, muss im Vordergrund stehen und nicht strategische Interessen externer Akteure, versteckt hinter opportunistisch genutzten oder - besser gesagt - missbrauchten Prinzipien. Die beiden favorisierten Modelle bieten die Möglichkeit, beide Aspekte - Minimierung des Leidens durch Selbstbestimmungsrecht sowie die Pflege des internationalen Rechts - miteinander zu harmonisieren. Eine unilaterale Anerkennungspolitik vorbei am UN-Sicherheitsrat würde hingegen einen völkerrechtlichen Supergau bedeuten. Der Westen sollte sich seine Lösungsoptionen genau überlegen, denn es steht weit mehr auf dem Spiel als die global betrachtet kleine und unbedeutsame Region Kosovo. Allein vor diesem Hintergrund existiert für den Westen schlichtweg kein Grund, den Forderungen der albanischen Seite vollständig nachzugeben oder sich durch Drohungen einschüchtern oder durch mitleidserweckende Attitüden lenken zu lassen. Das Kosovo ist auf Jahrzehnte von westlichen, d.h. vor allem aus Brüssel kommenden, Finanzspritzen abhängig. Die EU und die gesamte internationale Gemeinschaft sind die machtvolleren Akteure und sollten dementsprechend die Gestaltungskompetenz wieder an sich reißen. 